\title{
Multi-radio channel allocation in competitive wireless networks
}

\author{
Mark Felegyhazi, Mario Cagalj, Jean-Pierre Hubaux \\ EPFL - Switzerland \\ email: \{mark.felegyhazi,mario.cagalj,jean-pierre.hubaux\}@epfl.ch
}

\begin{abstract}
Channel allocation has been extensively studied in the framework of cellular networks, but the emergence of new system concepts, such as cognitive radio systems, bring this topic into the focus of research again. In this paper, we provide a formal analysis of the selfish multi-radio channel allocation problem using game theory. We conclude that in spite of the non-cooperative behavior of such devices, their channel allocation results in a Pareto- and system-optimal solution. Furthermore, we present a simple algorithm to achieve this efficient channel allocation. To the best of our knowledge, our paper is the first contribution to this important topic.
\end{abstract}

\section{Introduction}

Frequency Division Multiple Access (FDMA) is one of the widely used techniques that enable the simultaneous communication of several users on a given frequency band $[14,15]$. The basic principle of FDMA is to split up the available bandwidth to distinct sub-bands called channels. Assigning the radio transmitters to these channels is commonly referred to as the channel allocation problem ${ }^{1}$. Not surprisingly, an efficient channel allocation is a cornerstone of the design of existing wireless networks.

The channel allocation can be either fixed or dynamic, both approaches have advantages and drawbacks, depending on the considered scenario. Accordingly, there has been a significant amount of work that addresses both types of channel allocation in cellular networks. To study fixed channel allocation, most authors used graph coloring / labelling techniques (e.g., in [16]), whereas for dynamic channel allocation different solutions were developed (e.g. as presented in $[4,17])$. For a comprehensive survey on the topic, we refer the reader to [9].

1 In the literature, the terms channel assignment and frequency assignment are also used for the channel allocation problem.
Recently, several researchers have considered devices using multiple radios, notably in mesh networks. In the multi-radio communication context, channel allocation and access also became one of the crucial topics. Related work on multi-radio medium access includes $[1,2,13]$.

In all the related work cited so far, their authors assumed that the radio devices cooperate to achieve a high system performance. This assumption might not hold, as the users of these devices are usually selfish and they want to maximize their own performance without necessarily respecting the system objectives. Game theory provides a straightforward tool to study channel allocation in competitive wireless networks. A fixed channel allocation game was presented in [7] based on graph coloring. Furthermore, the authors of [12] present a selfish and a cooperative channel allocation protocol, and study their performance.

A complementary method for medium access, which is usually applied together with FDMA, is to use a protocol that enables Time Division Multiple Access (TDMA). This means that the devices coordinate their access to a particular channel over time. One widely used method is a distributed random access method called CSMA/CA [14]. Game theory has been applied to the CSMA/CA protocol $[6,10]$ and to the Aloha protocol [11].

In this paper, we present a game-theoretic analysis of fixed channel allocation strategies of devices using multiple radios. We assume that a channel access is solved on a particular channel and leave the study of joint channel allocation and medium access for future study. The work presented in this paper is a first step towards the deeper understanding of the non-cooperative behavior of selfish devices with multiple radios and is applicable in a broad context, notably in cognitive radio systems [8]. To the best of our knowledge, our paper is the first to address the problem of multi-radio channel allocation in competitive networks.

The paper is organized as follows. In Section 2, we introduce our system model along with a game-theoretic description of competitive channel allocation. Section 3 provides a comprehensive analysis of the channel allocation game. Finally, we conclude in Section 4. 


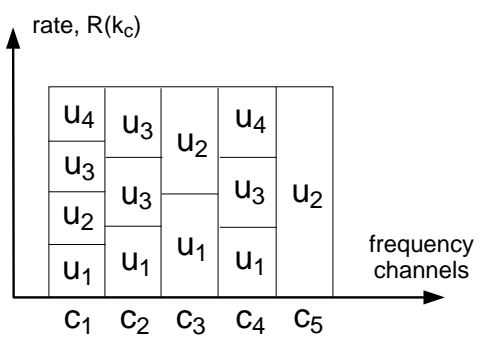

Figure 1. An example for a channel allocation with a constant rate function $R\left(k_{c}\right)$. Here $|\mathcal{N}|=4, k=4$ and $|\mathcal{C}|=5$.

\section{System Model}

We make the following assumptions with respect to the communication system. We assume an available frequency band divided into orthogonal channels of the same bandwidth using the FDMA method. We also assume that these channels have the same expected channel characteristics. We denote the set of available channels by $\mathcal{C}$. There exists a set of users $\mathcal{N}$, who want to access these channels. Each user owns a device equipped with $k \leq|\mathcal{C}|$ radio transmitters, all having the same communication capabilities. We assume that there is a mechanism that enables user devices to use multiple channels for their traffic at the same time (as it is implemented in [1] for example). We denote the number of radios of user $i$ using channel $c$ by $k_{i, c}$ for every $c \in \mathcal{C}$. For the simplicity of presentation, let us denote the set of channels used by user $i$ by $\mathcal{C}_{i}$, where $\mathcal{C}_{i} \subset \mathcal{C}$ and $0 \leq\left|\mathcal{C}_{i}\right| \leq k$. We further assume that there is no limitation on the number of radios per channel.

We formulate the multi-radio channel allocation problem as a non-cooperative game as follows. We define the strategy of user $i$ as its channel allocation vector:

$$
s_{i}=\left\{k_{i, 1}, \ldots, k_{i,|\mathcal{C}|}\right\}
$$

Hence, its strategy consists in defining the number of radios on each of the channels ${ }^{2}$. The strategy vector of all users defines the strategy matrix $S$, where the row $i$ of the matrix corresponds to the strategy vector of user $i$ :

$$
S=\left(\begin{array}{c}
s_{1} \\
\ldots \\
s_{|\mathcal{N}|}
\end{array}\right)
$$

Furthermore, we denote the strategy matrix except for the strategy of user $i$ by $S_{-i}$ :

2 Note that this number can be zero

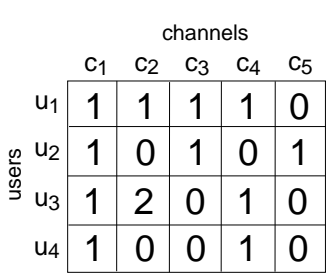

Figure 2. Strategy matrix of the example in Figure 1.

Figure 1 presents an example channel allocation, and Figure 2 presents the strategy matrix that corresponds to this example.

The total number of radios employed by user $i$ can be written as $k_{i}=\sum_{c} k_{i, c}$. Similarly, we can obtain the number of radios using a particular channel $k_{c}=\sum_{i} k_{i, c}$. Each user has a radio on channel $c_{1}$, but channel $c_{5}$ is occupied only by user $u_{2}$. User $u_{3}$ employs two radios on channel $c_{2}$ to get more bandwidth on that particular channel. Note that in Figure 1, we have $k_{u_{1}}=4, k_{u_{2}}=3, k_{u_{3}}=4$ and $k_{u_{4}}=2$, meaning that user $u_{2}$ and $u_{4}$ are not using all of their radios.

We assume that the users are rational and their objective is to maximize their utility in the network. We denote the utility of user $i$ by $U_{i}$. For simplicity, we assume that the user wants to maximize its total rate in the system and thus the utility function is the achieved bitrate. We leave the study of other utility functions for future work.

We assume that the total rate on channel $c$ is shared equally among the radio transmitters using that channel. This fair rate allocation is achieved for example by using a reservation-based TDMA schedule on a given channel. A similar result was reported by Bianchi in [3] for the CSMA/CA protocol using optimal backoff window values. Even if the radio transmitters are controlled by selfish users in the CSMA/CA protocol, they can achieve this fair sharing as shown in [6]. We further assume that the total available bitrate $R_{c}\left(k_{c}\right)$ on a channel $c$ (i.e., the sum of the achieved bitrate of all users on channel $c$ ) is a non-increasing function of the number of radios $k_{c}$ deployed on this channel. In fact $R_{c}\left(k_{c}\right)$ is independent of $k_{c}$ for a TDMA protocol and for the CSMA/CA protocol using optimal backoff window values [3]. In practice, the backoff window values used in the CSMA/CA protocol implementation (e.g., in the 802.11 standard) are not optimal; and due to packet collisions $R_{c}\left(k_{c}\right)$ becomes a decreasing function for $k_{c}>1$. Since we assume that channels have the same bandwidth and channel characteristics, the rate function does not depend on the channel and thus we can write that $R\left(k_{c}\right)$ for any channel $c \in \mathcal{C}$. If $k_{c}=0$, we define $R(0)=0$; note however that this case has no relevance in our model. 


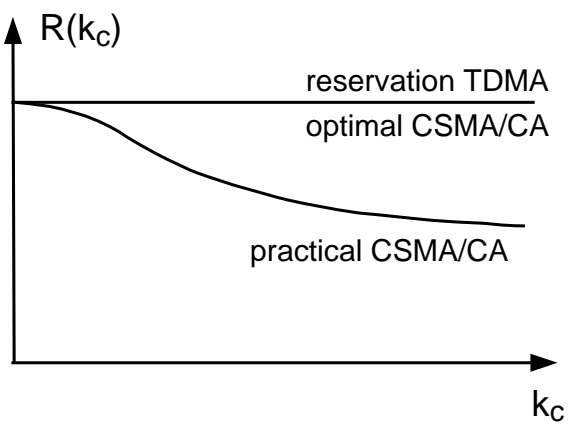

Figure 3. The total available rate $R\left(k_{c}\right)$ for different MAC protocols.

Figure 3 presents the total rate $R\left(k_{c}\right)$ as a function of the number of radios using channel $c$.

If user $i$ chooses to operate $k_{i, c}$ radios in a given channel, its rate can be written as $R_{i, c}=\frac{k_{i, c}}{k_{c}} \cdot R\left(k_{c}\right)$. Note that $R_{i, c}>0$ for all $c \in \mathcal{C}$, where $k_{i, c}>0$.

Thus, we can write the utility function for user $i$ as:

$$
U_{i}(S)=\sum_{c \in \mathcal{C}} R_{i, c}=\sum_{c \in \mathcal{C}} \frac{k_{i, c}}{k_{c}} \cdot R\left(k_{c}\right)
$$

\subsection{Game-Theoretic Preliminaries}

We assume that the transmitters reside in the same collision domain. We model the channel allocation problem with a single stage game, which corresponds to a fixed channel allocation among the users.

In order to study the strategic interaction of the users, we introduce some game-theoretic concepts:

Definition 1 (Nash Equilibrium - NE). The strategy matrix $S^{*}=\left\{s_{1}^{*}, \ldots, s_{|\mathcal{N}|}^{*}\right\}$ defines a Nash Equilibrium (NE), if for every user $i$ we have:

$$
U_{i}\left(s_{i}^{*}, S_{-i}^{*}\right) \geq U_{i}\left(s_{i}^{\prime}, S_{-i}^{*}\right)
$$

for every strategy $s_{i}^{\prime}$.

In other words, in a NE none of the users can unilaterally change its strategy to increase its utility. A NE solution is often inefficient from the system point of view. We characterize the efficiency of the solution by the concept of Pareto-optimality:

Definition 2 (Pareto-Optimality). The strategy matrix $S^{p o}$ is Pareto-optimal if for every user $i$ the following condition holds:

$$
U_{i}\left(S^{p o}\right) \geq U_{i}\left(S^{\prime}\right), \forall S^{\prime}
$$

This means that a Pareto-optimal channel allocation $S^{p o}$ cannot be improved upon without decreasing the utility of at least one player.

\section{Analytical Results}

In this section, we present our analytical results. It is straightforward to see that if the total number of radios is smaller than or equal to the number of channels, then a flat channel allocation, in which every channel is used by a single radio, is an equilibrium.

Fact 1. If $|\mathcal{N}| \cdot k \leq|\mathcal{C}|$, then any channel allocation, in which $k_{c}=1, \forall c \in \mathcal{C}$ is a Pareto-optimal NE.

For the remainder of the paper, we assume that $|\mathcal{N}| \cdot k>$ $|\mathcal{C}|$, hence the devices have a conflict during the channel allocation process. In the following lemmas, we express necessary conditions for a Nash Equilibrium. The first necessary condition shows that the users should use all of their radios.

Lemma 1. In a NE of the multi-radio channel allocation game, $k_{i}=k, \forall i$, meaning that each user is using all of its radios to communicate.

In the example presented in Figure 1, Lemma 1 does not hold for users $u_{2}$ and $u_{4}$. Hence, the example cannot be a NE.

Proof. We can prove the lemma by contradiction. Assume that there exists a NE, in which user $i$ uses only $k_{i}<k$ radios. As mentioned previously, in our model we assume that $k \leq|\mathcal{C}|$ and we assumed that in the $\mathrm{NE}\left|\mathcal{C}_{i}\right| \leq k_{i}<k$, thus we necessarily have $\left|\mathcal{C}_{i}\right|<|\mathcal{C}|$. This implies that there always exists a channel $c \notin \mathcal{C}_{i}$. If the user deploys an additional radio on this channel $c$, then she increases her utility due to the fact that $R_{i, c}>0$ for $k_{i, c}=1$. Hence, we have a contradiction and the original allocation cannot be a NE.

Let us now consider a NE strategy matrix in the multiradio channel allocation game denoted by $S^{*}$, where $s_{i}^{*} \in$ $S^{*}$ is the NE strategy of user $i$ (i.e., the i-th row of the matrix). Let us consider two arbitrary channels $b$ and $c$ in this NE strategy allocation. Without loss of generality, we assume that there are more radios using channel $b$, meaning that $k_{b} \geq k_{c}$, and denote their difference by:

$$
\delta_{b, c}=k_{b}-k_{c}
$$

Assume that user $i$ moves one of its radios from channel $b$ to $c$. Let us define the benefit of change, i.e. the difference in the utility of user $i$, as follows:

$$
\begin{aligned}
\Delta & =U_{i}\left(s_{i}^{\prime}, S_{-i}^{*}\right)-U_{i}\left(s_{i}^{*}, S_{-i}^{*}\right) \\
& =\frac{k_{i, b}-1}{k_{b}-1} \cdot R\left(k_{b}-1\right)+\frac{k_{i, c}+1}{k_{c}+1} \cdot R\left(k_{c}+1\right) \\
& -\frac{k_{i, b}}{k_{b}} \cdot R\left(k_{b}\right)-\frac{k_{i, c}}{k_{c}} \cdot R\left(k_{c}\right)
\end{aligned}
$$

We can show a second necessary condition for a NE. 
Lemma 2. If $k_{i, b}>0, k_{i, c}=0$ and $\delta_{b, c}>1$ for any user $i$, then $S^{*}$ is not a NE channel allocation.

This means that user $i$ has a benefit of moving one radio to a channel, where she has no radios if the difference of the number of radios deployed on the two channels exceeds one. In the example presented in Figure 1, Lemma 2 holds e.g. for user $u_{1}$ and the channels $b=c_{4}$ and $c=c_{5}$. Hence, the example cannot be a NE.

Proof. Assume that $S^{*}$ is a NE channel allocation. Suppose that user $i$ moves one of its radios from channel $b$ to $c$. Using the conditions in the lemma, we can write the benefit of change defined in (7) as:

$$
\begin{aligned}
\Delta & =\frac{k_{i, b}-1}{k_{b}-1} R\left(k_{b}-1\right)+\frac{1}{k_{c}+1} R\left(k_{c}+1\right)-\frac{k_{i, b}}{k_{b}} R\left(k_{b}\right) \\
& =\frac{k_{i, b}}{k_{b}-1} R\left(k_{b}-1\right)-\frac{1}{k_{b}-1} R\left(k_{b}-1\right) \\
& +\frac{1}{k_{c}+1} R\left(k_{c}+1\right)-\frac{k_{i, b}}{k_{b}} R\left(k_{b}\right)
\end{aligned}
$$

Let us notice that the sum of the first and last terms is always strictly greater than 0 , because $\delta_{b, c}>1$ implies that $k_{b}>1$. Hence, it is enough to investigate the sign of the sum of the two other terms. Using (6), we can rewrite the sum of the two middle terms as:

$\frac{R\left(k_{c}+1\right)}{k_{c}+1}-\frac{R\left(k_{c}-1\right)}{k_{c}-1}=\frac{R\left(k_{c}+1\right)}{k_{c}+1}-\frac{R\left(k_{c}+\delta_{b, c}-1\right)}{k_{c}+\delta_{b, c}-1}$

Due to the assumption $\delta_{b, c}>1$ and the non-increasing rate function $R(\cdot)$, we have:

$$
\frac{R\left(k_{c}+1\right)}{k_{c}+1}-\frac{R\left(k_{c}+\delta_{b, c}-1\right)}{k_{c}+\delta_{b, c}-1} \geq 0
$$

Hence, the benefit of change is positive and thus $S^{*}$ cannot be a NE. This contradiction concludes the proof.

Let us now derive the third necessary condition.

Lemma 3. If $k_{i, b}>1, k_{i, c}=0$ and $\delta_{b, c}=1$ for any user $i$, then $S^{*}$ is not a NE.

In the example presented in Figure 1, the conditions of Lemma 3 hold for user $u_{3}$ and the channels $b=c_{2}$ and $c=$ $c_{3}$. Hence, the example cannot be a NE.

Proof. We prove the lemma by contradiction. Assume that $S^{*}$ is a NE and there exists a user $i$ with $k_{i, b}>1, k_{i, c}=0$ and $\delta_{b, c}=1$. Assume that this user moves one of its radios from channel $b$ to $c$. Let us express the benefit of change (7) given the conditions of the lemma:

$$
\Delta=\frac{k_{i, b}-1}{k_{b}-1} \cdot R\left(k_{b}-1\right)+\frac{1}{k_{c}+1} \cdot R\left(k_{c}+1\right)-\frac{k_{i, b}}{k_{b}} \cdot R\left(k_{b}\right)
$$

Using $\delta_{b, c}=1$, we can reformulate this expression as follows:

$$
\Delta=\frac{k_{i, b}-1}{k_{b}-1} \cdot R\left(k_{b}-1\right)-\frac{k_{i, b}-1}{k_{b}} \cdot R\left(k_{b}\right)
$$

Note that (8) is always positive for $k_{b} \geq k_{i, b}>1$. Hence moving one radio is beneficial to user $i$ and $S^{*}$ cannot be a NE.

Suppose now that we have two channels $b$ and $c$ such that $k_{b}=k_{c}$. Assume that we have a user $i$ with $k_{i, b}>k_{i, c}>0$. Let us define the integer value $\gamma_{i, b, c}$ as:

$$
\gamma_{i, b, c}=k_{i, b}-k_{i, c}
$$

Using the value introduced above, we can derive the fourth necessary condition.

Lemma 4. If $\gamma_{i, b, c} \geq 2, k_{i, c}=0$ and $\delta_{b, c}=0$ for any user $i$, then $S^{*}$ is not a $N E$.

Proof. Assume that we have a NE $S^{*}$ in which $\gamma_{i, b, c} \geq 2$, $k_{i, c}=0$ and $\delta_{b, c}=0$ for user $i$. Assume that user $i$ moves one of its radios from channel $b$ to $c$ and let us express the benefit of change (7) as:

$$
\begin{aligned}
\Delta & =\frac{\gamma_{i, b, c}-1}{k_{c}-1} R\left(k_{c}-1\right)+\frac{1}{k_{c}+1} R\left(k_{c}+1\right)-\frac{\gamma_{i, b, c}}{k_{c}} R\left(k_{c}\right) \\
& =\gamma_{i, b, c}\left(\frac{R\left(k_{c}-1\right)}{k_{c}-1}-\frac{R\left(k_{c}\right)}{k_{c}}\right)-\left(\frac{R\left(k_{c}-1\right)}{k_{c}-1}-\frac{R\left(k_{c}+1\right)}{k_{c}+1}\right)
\end{aligned}
$$

Since the multiplier of $\gamma_{i, b, c}$ is always positive in the first term, we have a positive benefit of change (i.e., $\Delta>0$ ) if:

$$
\gamma_{i, b, c}>\frac{\frac{R\left(k_{c}-1\right)}{k_{c}-1}-\frac{R\left(k_{c}+1\right)}{k_{c}+1}}{\frac{R\left(k_{c}-1\right)}{k_{c}-1}-\frac{R\left(k_{c}\right)}{k_{c}}}
$$

After some simplifications, we get:

$$
\gamma_{i, b, c}>\frac{k_{c}\left(k_{c}+1\right) \cdot R\left(k_{c}-1\right)+k_{c}\left(k_{c}-1\right) \cdot R\left(k_{c}+1\right)}{\left(k_{c}^{2}-1\right) \cdot R\left(k_{c}\right)+k_{c}\left(k_{c}+1\right) \cdot R\left(k_{c}-1\right)}
$$

Note that all terms in the expression are strictly positive, thus we can increase its value as follows:

$$
\begin{aligned}
\gamma_{i, b, c} & >\frac{k_{c}\left(k_{c}+1\right) \cdot R\left(k_{c}-1\right)+k_{c}\left(k_{c}-1\right) \cdot R\left(k_{c}+1\right)}{k_{c}\left(k_{c}+1\right) \cdot R\left(k_{c}-1\right)} \\
& =1+\frac{k_{c}\left(k_{c}-1\right) \cdot R\left(k_{c}+1\right)}{k_{c}\left(k_{c}+1\right) \cdot R\left(k_{c}-1\right)}
\end{aligned}
$$

Let us notice that the fraction is strictly positive, but smaller than one. Hence, we can write that $\Delta>0$ for:

$$
\gamma_{i, b, c} \geq 2
$$

This implies that moving the radio from one channel to another is beneficial for user $i$ and therefore $S^{*}$ cannot be a NE. 
Let us now consider a channel allocation $S$ and let us define the set of channels $C_{\max }$ with the maximum number of radios (i.e., where $b \in C_{\max }$ has $k_{b}=\max _{l \in \mathcal{C}} k_{l}$ ). Similarly, let us define the set of the least loaded channels $C_{\text {min }}$, where $c \in C_{\text {min }}$ has $k_{c}=\min _{l \in \mathcal{C}} k_{l}$. We denote the set of the remaining channels by $C_{r e m}$. In Figure 1, $C_{\max }=\left\{c_{1}\right\}, C_{\min }=\left\{c_{5}\right\}$ and $C_{\text {rem }}=\left\{c_{2}, c_{3}, c_{4}\right\}$.

Using Lemmas 1, 2, 3 and 4 , we conclude on a fifth necessary condition.

Proposition 1. In a NE $S^{*}$ in the multi-radio channel allocation game, we have $\delta_{b, c} \leq 1$ for all $b, c \in \mathcal{C}$.

Proof. In the proof, we show that if there exist $b, c \in \mathcal{C}$ such that $\delta_{b, c}>1$ then at least one of the lemmas apply and hence $S^{*}$ cannot be a NE.

Let us now consider two channels $b$ and $c$ such that $b \in$ $C_{\max }$ and $c \in C_{\min }$.

- If there exists user $i$ such that $k_{i, b}>0$ and $k_{i, c}=0$, then Lemma 2 applies.

- If for all users $i$ with $k_{i, d}>0$, we have $k_{i, c}>0$, then there must be at lease one user $j$ such that $k_{j, b}>1$ due to the assumption that $\delta_{b, c}>1$ (i.e., user $j$ has two or more radios on channel $b$ ). Due to the assumption $k \leq \mathcal{C}$, there must be a channel $a$ such that $k_{j, a}=0$. If $a \in C_{\text {min }}$, then Lemma 2 applies and if $a \in C_{\text {rem }}$, then Lemma 3 holds.

- Finally we will prove that such a case cannot exist, where none of the above conditions apply. Since the above cases do not apply, user $j$ has $k_{j, c}>0$ and $k_{j, d}>0$ for all $c \in C_{\text {min }}$ and $d \in C_{\text {rem }}$ (i.e., user $j$ has a radio on every channels that belong to the sets $C_{\min }$ and $C_{\text {rem }}$; whereas it has two radios on at least the channel $b$ ). Hence, we have the following condition on the number of radios deployed by user $j$ :

$$
k=k_{j} \geq\left|C_{\text {min }}\right|+\left|C_{r e m}\right|+2
$$

Furthermore, let us consider another user $u$ with $k_{u, c}=0$. Since $c \in C_{\text {min }}$, we know that such a user exists. Clearly, then we must have $k_{u, b}=0, \forall b \in$ $C_{\max }$ as well, otherwise Lemma 2 would apply to user $u$. Furthermore, user $u$ cannot have more than one radio on any channel $a \in C_{r e m}$, because then Lemma 3 would apply. Similarly, user $u$ can have at most one radio per channel for channels $a \in C_{\text {min }}$ and $a \neq c$, otherwise the conditions of Lemma 4 are violated. We can write a condition on the number of radios user $u$ deploys:

$$
k=k_{u} \leq\left|C_{r e m}\right|+\left|C_{\text {min }}\right|-1
$$

From Lemma 1, we have that $k_{j}=k_{u}=k$. Since $\left|C_{\text {min }}\right|$ and $\left|C_{\text {rem }}\right|$ are non-negative numbers, the impossibility of this case follows from the inequalities (10) and (11).

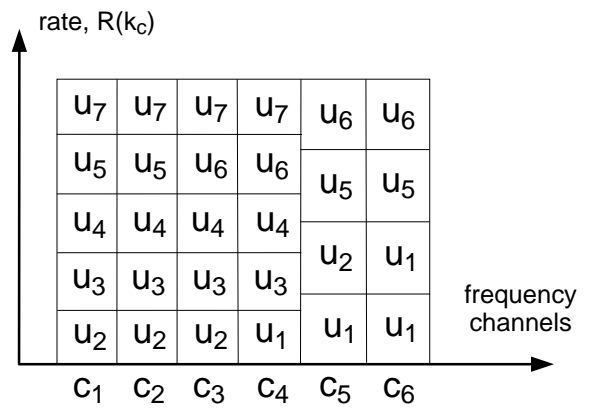

Figure 4. An example for a NE channel allocation. Here $|\mathcal{N}|=7, k=4$ and $|\mathcal{C}|=6$. Note that user $u_{1}$ belongs to the group of users which have an exception in the second condition.

This concludes the proof.

Combining these results, we can establish a set of necessary and sufficient conditions for the NE.

Theorem 1. Assume that we have $|\mathcal{N}| \cdot k>|\mathcal{C}|$. Then a channel allocation $S^{*}$ is a NE iff the two following conditions hold:

- $\delta_{b, c} \leq 1$ for any $b, c \in \mathcal{C}$ and

- $k_{i, c} \leq 1$ for any $b, c \in \mathcal{C}$ and $i \in \mathcal{N}$ except for users $j$ with $\nexists c \in C_{\text {min }}$ such that $k_{j, c}=0$. For such a user $j$, the second condition changes as follows: $k_{j, c} \leq 1$ if $c \in C_{\max }$ and $\gamma_{i, a, c} \leq 1$ for any channel $a, c \in C_{\min }$.

An example of a NE channel allocation is shown in Figure 4, where the second condition of the theorem has an exception for user $u_{1}$. Figure 5 presents an example with no exception on the second condition for any user.

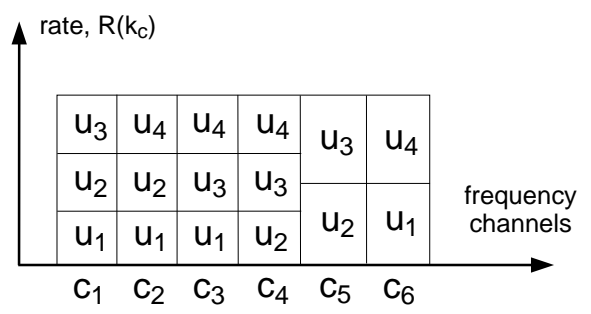

Figure 5. A NE channel allocation with no exception of the second condition. Here $|\mathcal{N}|=$ $4, k=4$ and $|\mathcal{C}|=6$. 
Proof. Let us show that the above conditions are necessary. Proposition 1 established that the first condition is necessary. Lemmas 2, 3 and 4 make the second condition necessary.

Now we prove that these conditions are sufficient as well. According to the first condition of the theorem, the difference between the number of radios on any two channels cannot be more than one, thus the set $C_{r e m}$ does not exist. This also means that any change consists in moving some radios from channels in $C_{\max }$ to channels in $C_{\min }$. Consequently, the moves can be considered separately.

Let us thus consider the moving of one radio from a channel $b \in C_{\max }$ to a channel $c \in C_{\min }$ which results in a strategy $s_{i}^{\prime}$ for user $i$. Substituting $\gamma_{i, b, c}=k_{i, b}-k_{i, c} \leq 1$, we can write the benefit of change expressed in (7) as follows:

$$
\begin{aligned}
\Delta & =\frac{k_{i, c}+\gamma_{i, b, c}-1}{k_{c}} R\left(k_{c}\right)+\frac{k_{i, c}+1}{k_{c}+1} R\left(k_{c}+1\right) \\
& -\frac{k_{i, c}+\gamma_{i, b, c}}{k_{c}+1} R\left(k_{c}+1\right)-\frac{k_{i, c}}{k_{c}} R\left(k_{c}\right) \\
& =\left(\gamma_{i, b, c}-1\right)\left(\frac{R\left(k_{c}\right)}{k_{c}}-\frac{R\left(k_{c}+1\right)}{k_{c}+1}\right)
\end{aligned}
$$

Note that second factor is always positive and hence the difference is non-positive for $\gamma_{i, b, c} \leq 1$. For this value, the strategy matrix $S^{*}$ defines a NE.

Theorem 1 establishes an interesting property about NE: In fact, all NE channel allocations achieve load-balancing over the channels in $\mathcal{C}$. In the next theorem, we will show that allowing selfish channel allocation over a wide band of frequencies results in an efficient spectrum utilization.

Theorem 2. Assume that we have $|\mathcal{N}| \cdot k>|\mathcal{C}|$. Then any $N E$ channel allocation $S^{*}$ is Pareto-optimal.

Proof. The proof is straightforward. In a NE channel allocation $S^{*}$ we have $k_{c}>0$ for each $c \in \mathcal{C}$. Note that in $S^{*}$, the sum of the utility of all users $U_{\text {total }}=\max _{i} \sum_{i} U_{i}$ which implies the Pareto-optimality.

One can notice that the Pareto-optimal NE is also system optimal, because it maximizes the total rate achieved by all users.

We can use the following simple algorithm to achieve one of these Pareto-optimal NE. Note that this algorithm follows from the extensive-form representation [5] of the channel allocation game and hence relies on the sequential action of the players. We emphasize that this algorithm is centralized, because it needs a coordination between the players to determine the order of allocating their radios. The development of a distributed implementation is an important part of our ongoing work.

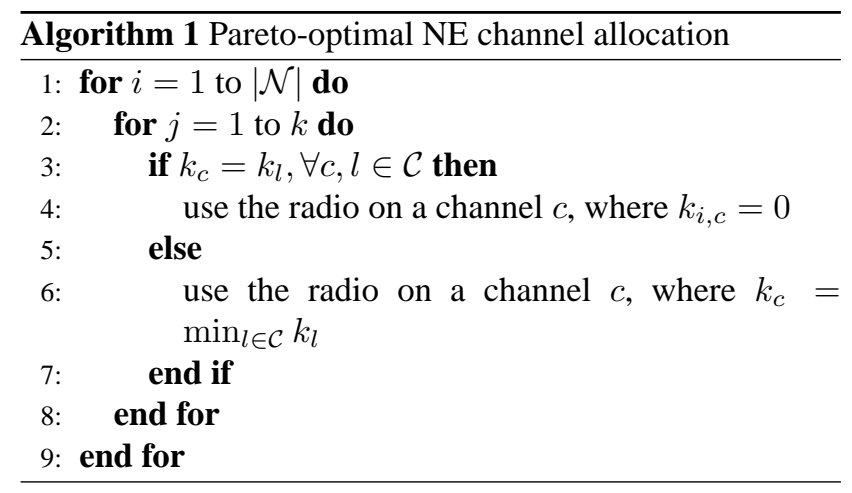

\section{Conclusion}

In this paper, we have considered the problem of competitive channel allocation among devices using multiple radios. Our main conclusion is that in spite of the non-cooperative behavior of such devices, their Nash equilibrium channel allocation achieves load-balancing and is system-optimal. This solution is characterized by the fact that the devices occupy the available channels almost evenly. Furthermore, we have provided a simple to achieve this efficient channel allocation.

\section{References}

[1] A. Adya, P. Bahl, J. Padhye, A. Wolman, and L. Zhou. A multi-radio unification protocol for IEEE 802.11 wireless networks. In Proc. of Broadnets'04, pages 344-354, 2004.

[2] M. Alicherry, R. Bhatia, and L. (Erran) Li. Joint channel assignment and routing for throughput optimization in multiradio wireless mesh networks. In Proc. of Mobicom'05, pages 58-72, Aug. 28-Sep. 22002.

[3] G. Bianchi. Performance analysis of the IEEE 802.11 distributed coordination function. IEEE Journal on Selected Areas in Communication, 18(3), March 2000.

[4] M. M. L. Cheng and J. C. I. Chuang. Performance evaluation of distributed measurement-based dynamic channel assignment in local wireless communications. IEEE Journal on Selected Areas in Communications, 14(4):698-710, May 1996.

[5] D. Fudenberg and J. Tirole. Game Theory. MIT Press, 1991.

[6] M. Cagalj S. Ganeriwal, I. Aad, and J.-P. Hubaux. On selfish behavior in CSMA/CA networks. In Proceedings of the IEEE Conference on Computer Communications (INFOCOM '05), March 13-17 2005.

[7] M. M. Halldórsson, J. Y. Halpern, L. (Erran) Li, and V. S. Mirrokni. On spectrum sharing games. In Proceedings of the 23rd annual ACM symposium on Principles of Distributed Computing (PODC 2004), pages 107-114, July 25-28 2004.

[8] S. Haykin. Cognitive radio: Brain-empowered wireless communications. IEEE Journal on Selected Areas in Communications (JSAC), 23(2):201-220, Feb. 2005.

[9] I. Katzela and M. Naghshineh. Channel assignment schemes for cellular mobile telecommunication systems: a compre- 
hensive survey. IEEE Personal Communications, 3(3):1031, Jun 1996.

[10] J. Konorski. Multiple access in ad-hoc wireless LANs with noncooperative stations. In Proceedings of Networking 2002, pages 1141-1146, 2002.

[11] A. B. MacKenzie and S. B. Wicker. Stability of multipacket slotted aloha with selfish users and perfect information. In Proceedings of the IEEE Conference on Computer Communications (INFOCOM '03), March 30 - April 32003.

[12] N. Nie and C. Comaniciu. Adaptive channel allocation spectrum etiquette for cognitive radio networks. In Proceedings of IEEE Dynamic Spectrum Access Networks (DySPAN'05), Nov. 2005.

[13] A. Raniwala and T.-C. Chiueh. Architecture and algorithms for an IEEE 802.11-based multi-channel wireless mesh network. In Proceedings of the IEEE Conference on Computer Communications (INFOCOM '05), March 13-17 2005.

[14] T. S. Rappaport. Wireless Communications: Principles and Practice (2nd Edition). Prentice Hall, 2002.

[15] M. Schwartz. Mobile Wireless Communications. Cambridge Univ. Press, 2005.

[16] J. van den Heuvel, R. A. Leese, and M. A. Shepherd. Graph labeling and radio channel assignment. Journal of Graph Theory, 29:263-283, 1998.

[17] J. Zander and H. Eriksson. Asymptotic bounds on the performance of a class of dynamic channels assignment algorithms. IEEE Journal on Selected Areas in Communications (JSAC), 11(6):926-933, Aug 1993. 